\title{
Analyzing the Important Factors for Cloud Service Provider Selection among the IT firms in Turkey
}

\author{
Asst. Prof. Dr. Ediz Şaykol (Beykent University, Turkey) \\ Ayşe Seçil Başer (Beykent University, Turkey) \\ Assoc. Prof. Dr. Kazim Sari (Beykent University, Turkey)
}

\begin{abstract}
Cloud computing, as being one of the trendy topics, provides mechanisms for the companies to meet all the information technology and management requirements at a desired performance level with reduced upfront investments. From the economical perspective, it creates a positive impact by helping them focus on their own business. With this positive impact, it causes a huge increase on the number of user firms, and many service providers try to provide solutions based on the user firms' needs. There are studies on identifying the factors that are used in selecting cloud service solutions in the literature. Having examined the pivotal points among the Information Technology (IT) sector in Turkey, we conducted a survey among the managers of various firms to gather information about their cloud service provider and the factors that provision their choice. Since that decision is very strategic, and once decided it is very hard to alter, it is worth analyzing the set of factors that are used in the survey further in order to identify the most important ones and help new companies for their selection. We provide statistical evaluations based on the results of the survey in this study.
\end{abstract}

\section{Introduction}

It is quite impossible to remain indifferent to technological innovations. These innovations on Information Technology (IT) world not only affect itself but also affect the other sectors, like a domino tile effect as well. With the progress of the information technologies, the IT sector becomes an important part and an indispensable identity of our lives. In today's world not being able to reach information; communication problems between themselves or not being up to news has been perished. In order to be able to have a more effective working strategy, companies/business enterprises have started to look for new horizons and technologies. The old servers which have not been favoured and left behind the technology race has long been replaced with virtual technologies. At the same time the demand and attention to virtual technologies have enabled business enterprises which are in a constant competition with other enterprises to keep the upper hand. This means whoever uses the technological advancements more actively and effectively, starts the competition a few steps ahead.

Even though the term "Cloud Computing" has been more popular than ever, the real functionality of cloud computing is not thoroughly known (Armbrust et al., 2009). This issue is caused by business enterprises who are using the cloud computing systems passing over the main key questions. The key questions should be "what am I using cloud computing for, what do I need". After identifying the answers to these key questions, cloud computing providers can custom fit the system to the end users' needs. With the perfect custom fit for the needs of the supply and demand; business enterprises can easily get the services for their needs, pay per usage, change the content of the services when they need and so forth decrease the costs with flexible cloud services. Thus it is obvious that cloud computing has a lot to offer to business enterprises. The statistical study of the survey for this paper aims to enlighten which of these elements are more demanded by the business enterprises. Via these results, business enterprises which decide to use cloud computing but do not know where to start can have an opinion by analyzing the other enterprises who are in the same sector or same scale using cloud computing. The main goal of this paper to shine a mirror on the usage of cloud computing and service providers among the IT firms in Turkey.

In this paper, some preliminary information is given in Section 2 for those who are unfamiliar to cloud computing terms at all. Section 3 gives brief examples of previous academic works and Section 4 is showing the main purpose of the paper which is the survey that obtained to SME's (Small-Medium Enterprises). Section 5 states statistical analysis based on the survey and finally, Section 6 concludes the paper.

\section{Preliminaries on Cloud Computing}

Cloud computing concept can differ in definitions but the main purpose is without any obstacles on locations reaching the data from anywhere and collaboration with multiple teams. Cloud computing is not a product that end users have but a service that meet the needs of the end user. The platform enables the sharing of information via internet from computers or other devices from anywhere. The most basic but the most extensive definition of cloud computing is controlling the needs of computers like hard disks, software and hardware from a remote location. Also it enables to reach the photographs, documents, media files from other computers. The decision of which of these data will be accessible is done by the service model which has been purchased.

The level of benefit from cloud computing can be categorized under three different service models (Saykol, 2014). The most fundamental cloud computing service IaaS provides virtual servers, operations, storage and 
bandwidths for developers and other cloud computing services. This service model which shows the cluster architectures most effective examples gives and undertaking to high accessibility ratios and back-ups. It is the platform of the system administrator.

Just like Iaas and SaaS which are providing developers as a part of service preparation process; PaaS provides operating systems, database, programming platform and runtime services. It provides the all of the sophisticated medium for the programme to run written by the developer. SaaS is the end user platform. The software is served to end user on this platform. This platform doesn't need extra processes, book value or configuration. It is ready to use and accessible from everywhere. It also provides continuity and data security. Figure 1 shows these three cloud computing service models.

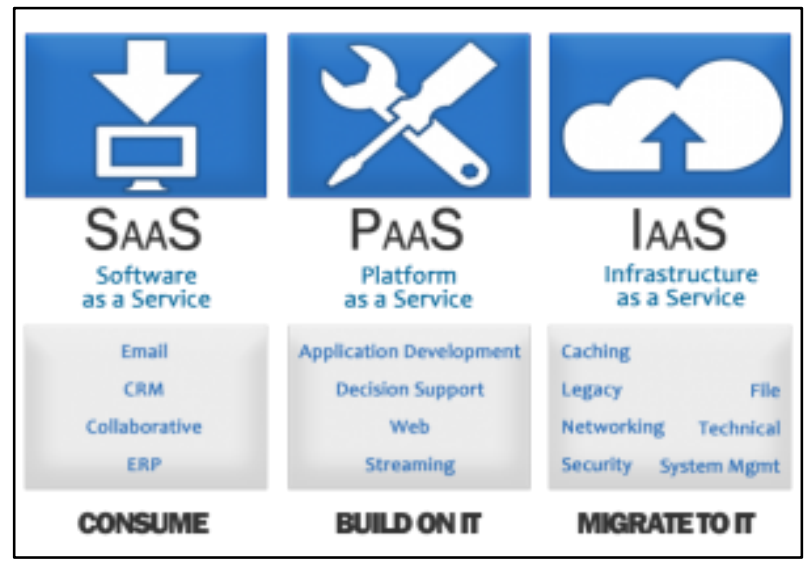

Figure 1. Cloud Computing Service Models Source: (Consultia, 2015).

\section{Literature Survey on Cloud Computing Criteria}

The cloud computing technologies offers the end users and enterprises important gains at the recent years. These technologies anticipate the manageable use of services which are bounded in a multilayered structure offers less book values and more efficient services.

For the last five years the academic researches on cloud computing has reached a number of 10.000 worldwide. The local and global studies subjects are provided below.

(Höfer and Karagiannis, 2011), has searched the building blocks of cloud computing and compared them with other traditional technologies. The study focused on the innovations that are brought by the cloud computing technologies from past to date.

(Kossman and Kraska, 2010) has done researches on book values of cloud computing systems and their costs to enterprises. They have touched upon the correlation between the services provided and usage ratios, also the costs of having these services.

(Pelletier, 2009) chose to identify how to pick the road to import services avoiding heavy costs for end-users or enterprises. Cloud computing has many meanings for end users or enterprises. To explain it simply cloud computing means to reach the applications over the internet in the easiest and fastest way for users. This model which is independent from the user's location provides reaching the datas or applications.

(Svantesson and Clarke, 2010) has researched on the risks that can be faced on using cloud computing especially the data security.

(Barret and Kipper, 2010) has studied the correlation between the virtual technologies and cloud computing and emphasized the role of virtual technologies in cloud computing.

(Issarny et al., 2011) has studied the service based approach and the effects of this approach on the internet in the future.

(Knorr and Gruman, 2008) has researched the cloud based services and the services between the cloud layers.

(De Roure, 2010) has done a research on e-science and web technologies' importance on present-day. In this study the infrastructure that are used to provide the web services to the users have been evaluated.

(Getov and Srinivasan, 2011) has emphasized the services that are provided by the cloud computing service providers.

(Ortiz, 2011) focused on the standardization issue of the cloud Technologies. The study emphasized on the disability of different virtualization technologies' compatibility and coordination between each other. Among this, the comparative analysis between traditional security solutions and cloud computing security has been made. 
Of course it is widely acknowledged that one of the main problems with cloud computing solutions is security. The confidentiality and continuity of the services provided are a big deal for end-users and enterprises. At this point (Garber, 2011) has studied the defined security gaps and risks of the cloud computing technologies and made some conclusions for these issues.

Korkmaz (2010) has focused on the data security, just like (Garber, 2011), legal regulations and service continuity of the cloud computing technologies from Turkey's perspectives.

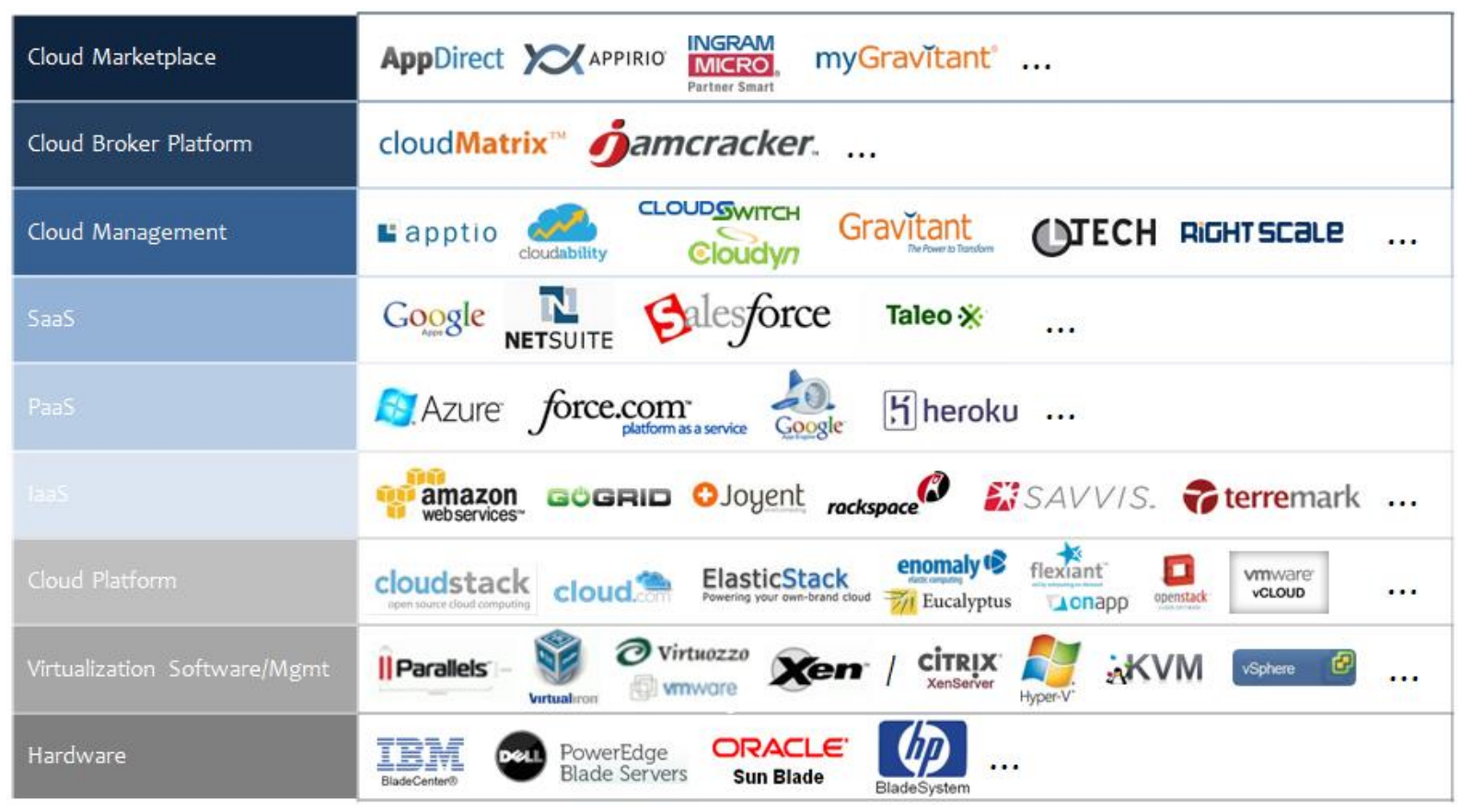

Figure 2. A Set of Cloud Service Providers Based On Service Models Source: (Jackson, 2014).

\section{The Survey to Analyze Cloud Service Providers}

The aim of this survey is to make a statistical analysis for the enterprises whom will benefit from the cloud computing systems especially Small and Medium Enterprises' (S.M.E). We also aim to find the most important factors for the selection of cloud service provider and shine a light on which service is chosen by which priority, by the enterprises that are in Turkey's informatique sectors. Figure 2 gives a set of cloud service providers globally around the world based on the service model they provide.

The survey has been made by the help of 57 volunteers on 21 informatique criteria. The participants have been asked to fill the questionnaire made up by 21 informatique criteria by the order of importance. The most important criteria would be number one whereas the least important criteria was 10 . The participants have also been asked to name the cloud service providers that they have been working with.

The participants are aged between 20-40, either the company owner or the employee of the company whom has a keen understanding on cloud computing. Some of the participants has long been with working with cloud computing systems whereas the others are quite new on these systems.

This paper has been prepared as a continuation of (Saykol and Avc1, 2015), which argues that benefiting from cloud computing services has a win-win effect on both customers and service providers, following a medium established in (Saykol, 2014).

Keeping in mind that cloud computing is in every section of our lives nowadays, taking this matter on an enterprise base; we can see that not only large organizations but in fact S.M.E.'s can also benefit from these services. So this survey has included S.M.E.'s. Due to the requests of the participants the names of the participants have been concealed.

\section{Statistical Analyses of the Survey}

The participants have been asked to complete the survey from most important to least important in an ascending manner. Accordingly, a numeric value is given to each criteria. Namely, the most important criteria was numbered as 10 and least important criteria is numbered as 1 . After that each criteria's mean values and standard deviation values has been calculated as shown in Figure 3 and Figure 4. 
After analyzing the mean values of the criteria for 57 participants, it is observed that expenditure is the most important criterion. This outcome has been expected since the main feature of cloud computing is lowering the costs for the enterprises.

Even though the most contentious and open to enhancement feature of the cloud computing is security; our survey has showed us that our participants have another priority. Since that in our literature review and researches the main drawback issue was security and in our survey the participants prioritized it; we have contacted with the participants.

The answers given by the participants were satisfying:

- The prior systems that they were using for data storage, system administration, performance enhancing were compromising the data security if there were no back-up units; whereas the data stored on the cloud servers has automatically serves a back-up and also these back-ups were accessible from anywhere.

- The enterprises who are working with professional cloud computing service providers has a 24/7 hotline with the provider thus help and proper guidance was in reach.

- In case of a problem caused in their prior systems data centers the enterprises had to ask from another company for maintenance and restoration whereas with cloud computing service providers they only have one interlocutor

In the light of these information given by the participants, customers feel safer in the hands of professional cloud computing service providers and that is why they are choosing to work with these providers.

The following criteria is performance and data accessibility. The participants also stated that they were satisfied with the service providers and the quality of performance. Also being able to access the data 24/7 without any locational limitations was another important criteria for the customers.

The first breaking point that is determined amongst the participants was virtualization. Even though participants have stated that the virtualization of the software and hardware resources was important; the participants dissented from each other about the criteria. The statistical analysis shown Figure 5 indicate that virtualization criteria are number one on standard deviation value.

Another element that draws our attention was pay as you go and services criteria were rated lower than we expected. Thus we can say that customers were choosing a package deal with a fixed price rather than pay per usage deals.

Even though one of the most important feature of cloud computing systems is "services" provided; to deduct that these services are extensively used by our participants would be wrong; due to the demographic distribution of the scales of the enterprises favors in S.M.E's. These S.M.E's are mainly SaaS users which provides limited authorization of control. In this case these enterprises probably know very little about the extent of the services.

The least important features rated by the participants were API and AJAX compatibility. We have identified that the main cause of this score for these features arises from the lack of knowledge of the participants for what these words stand for even though they are using these features. Also the participants who stated that they know about API and AJAX compatibility services stated that these features are a must have on cloud computing systems so forth there's no need to prioritize these features.

Figures 3 and 4 give the mean value analysis and standard deviation results, respectively for the survey carried out during this study.

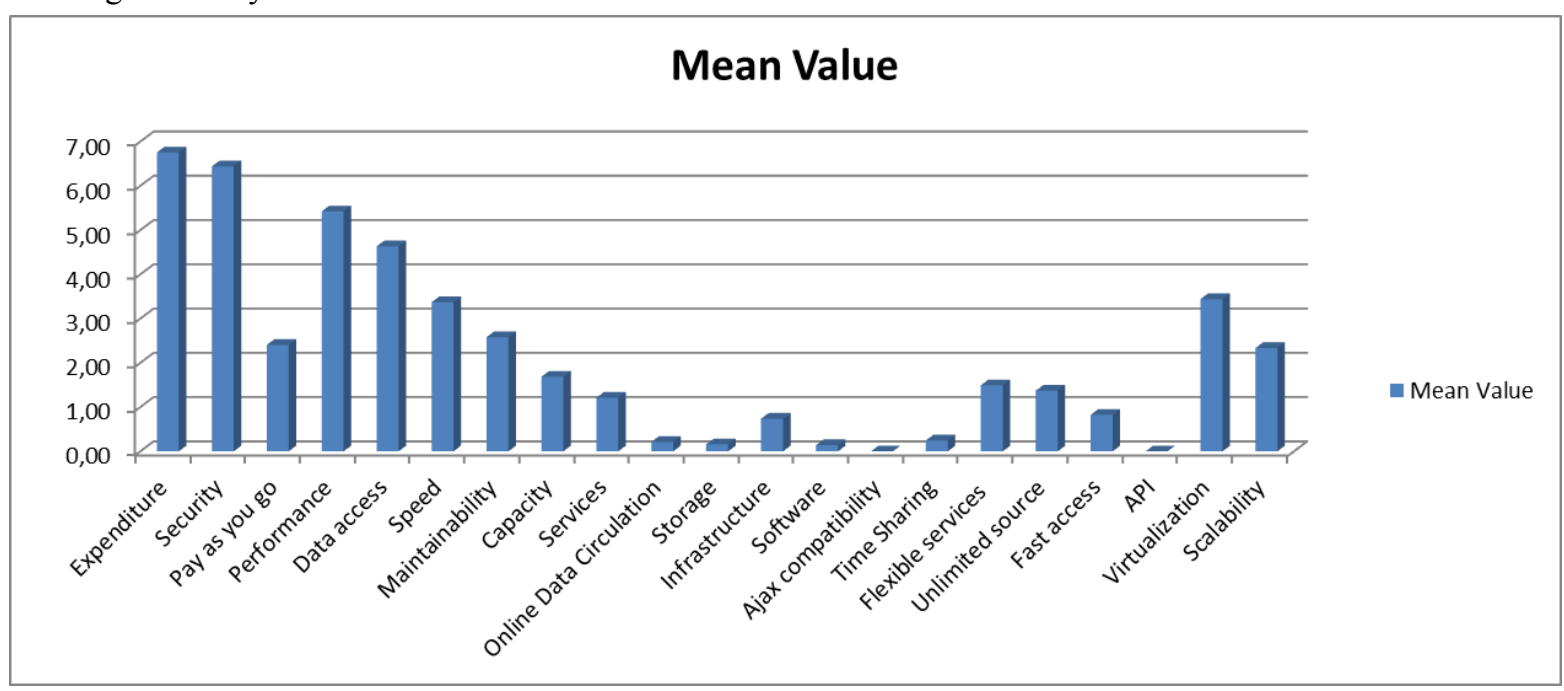

Figure 3. The Mean Value Analysis of the Criteria Used in The Survey. 


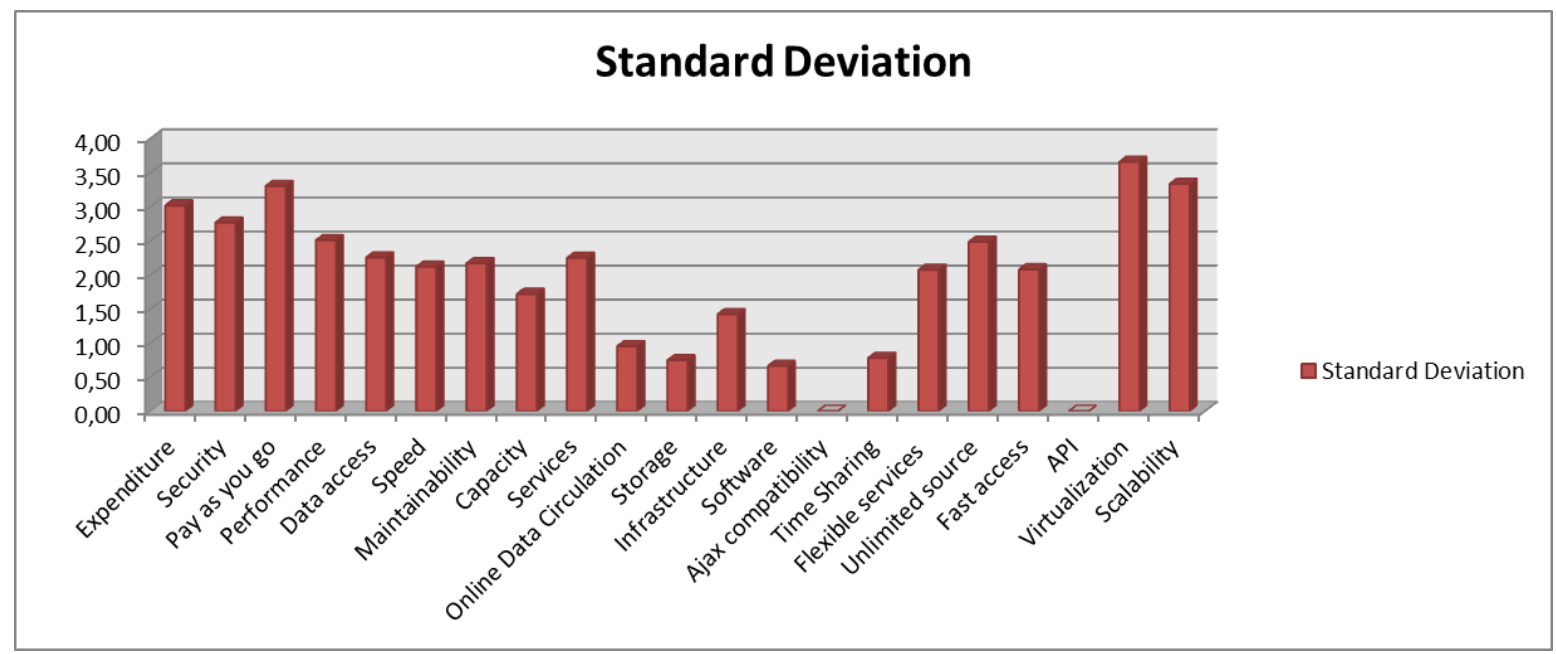

Figure 4. The Standard Deviation of the Criteria Used in The Survey.

Additionally, coefficient of variation for each criterion is also calculated in Figure 5 to find out the confidence interval. This is performed to understand if there is a consensus about the scores of each criterion. This calculation gives the percentage difference of standard deviation to mean value.

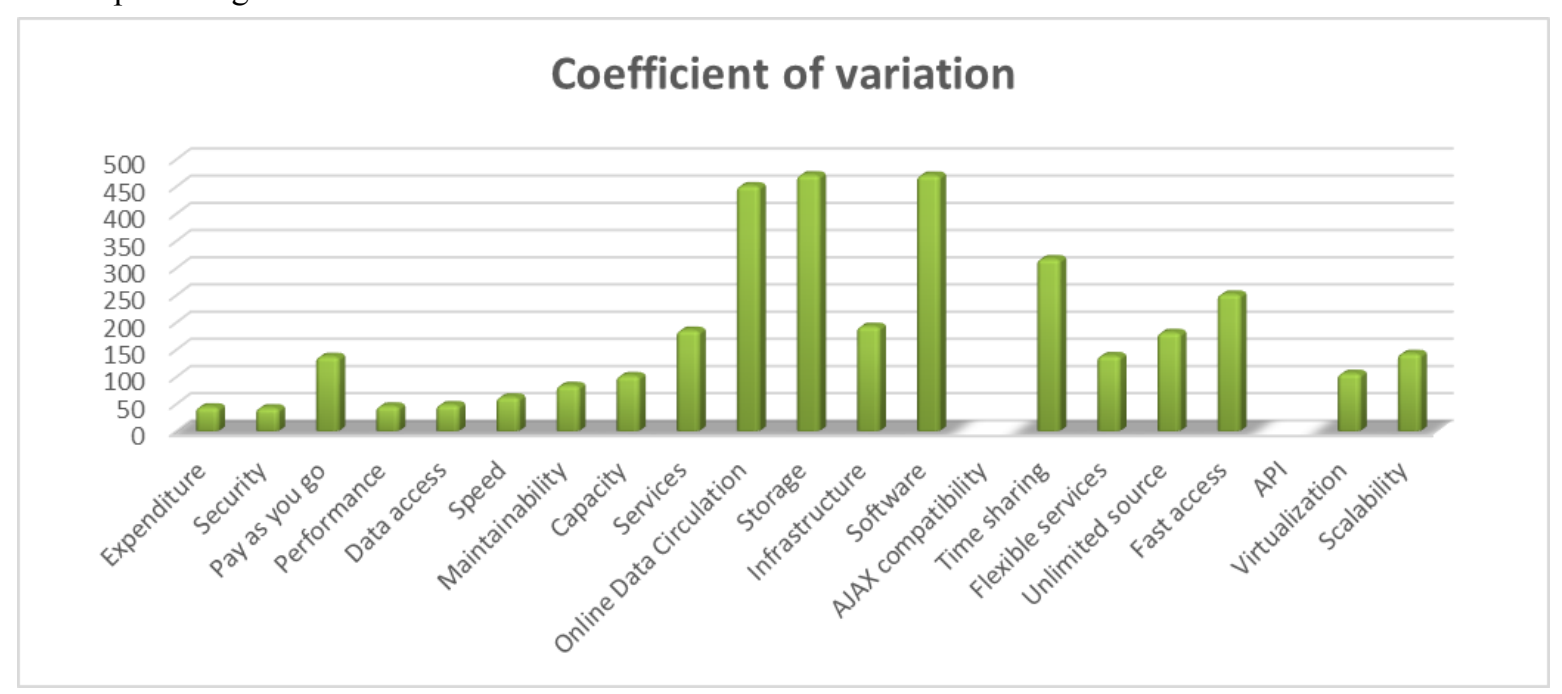

Figure 5. The Coefficient of Variation of the Criteria Used in The Survey.

It can be said from Figure 5 that expenditure, security, performance, data access are the criteria that all most all participants are in consensus while there are some different opinions for the remaining criteria.

\section{Conclusion}

Cloud computing expresses offering the technologies that originate from the past as a service. These services are offered via multiple layers that cloud computing services can be used in. With the invention and increasing usage of the internet, the usage of the cloud computing services by individuals or enterprises has increased as well. By accessing these services for affordable prices and lowering the IT costs; enterprises has profited in acceptable amounts and became more efficient. For the past five years the increasing usage of cloud computing has been more preferred by the S.M.E.'s. The enterprises who choose dynamic service providers data centers over bulky data centers can save from maintenance, personnel and licensing costs.

The usage of cloud computing systems which are providing flexible, scalable and modifiable for the needs of usage for the enterprises is also important for the national energy savings. The aim is to increase the efficiency of the enterprises by removing the unnecessary data center costs and paying per usage. Via accessing and sharing the data from everywhere; a more efficient labour and business benefits can be achieved. For these features cloud computing is seen as the technology of the future.

In this study, an empirical work is performed to reveal the most important factors for the selection of cloud service provider. By this way, it is made clear the managers' decision approach to the cloud service providers. Thus, this study will form a base for the further studies to construct a decision model for this purpose. 


\section{References}

- $\quad$ Armbrust, Michael, Fox, Armando, Griffith, Rean, Joseph, Anthony D., Katz, Randy H., Konwinski, Andrew, Lee, Gunho, Patterson, David A., Rabkin, Ariel, Stoica, Ion and Zaharia, Matei, 2009, "Above the Clouds: A Berkeley View of Cloud Computing", UCB/EECS-2009-28, http://www.eecs.berkeley.edu/Pubs/TechRpts/2009/EECS-2009-28.html.

- Barrett, Diane, Kipper, Gregory, 2010, Virtualization and Forensics: A Digital Forensic Investigator's Guide to Virtual Environments, Elsevier Inc, MA, USA.

- Consultia, 2015, "Cloud computing service models", http://www.consultia.co/choosing-your-right-cloud/.

- De Roure, David, 2010, "e-Science and the Web", IEEE Computer, Volume 43, Issue 5, 90-93, May.

- Garber, Lee, 2011, "News Briefs", IEEE Computer, Volume 44, Issue 7, 17-19, July.

- Getov, Vladimir, Srinivasan, Savitha, 2011, "From invisible grids to smart cloud computing", Proceedings of Euro-Par 2010 Parallel Processing Workshops, Lecture notes in Computer Science Volume 6586, Springer, 263-270.

- Höfer, C.N., Karagiannis, G., 2011, “Cloud Computing Services: Taxonomy and Comparison”, Journal of Internet Services and Applications, Volume 2, Issue 2, 81-94.

- Issarny, Valérie, Georgantas, Nikolaos, Hachem, Sara, Zarras, Apostolos, Vassiliadist, Panos, Autili, Marco, Gerosa, Marco Aurelio, Ben Hamida, Amira, 2011, "Service-oriented middleware for the future internet: state of the art and research directions", Journal of Internet Services and Applications, Volume 2, Issue 1, 23-45, Springer-Verlag.

- Jackson, Kevin, 2014, Forbes, http://blogsimages.forbes.com/kevinjackson/files/2014/06/cloudtechspectrum_vendors_v211.png.

- Knorr, Eric, Gruman, Galen, 2008, "What cloud computing really means", InfoWorld Magazine (online), http://www.infoworld.com/article/2683784/cloud-computing/what-cloud-computing-really-means.html.

- Korkmaz, Yakup, 2010, "Bulut Bilişim Risk Değerlendirmesi”, http://www.bilgiguvenligi.gov.tr/guvenlikteknolojileri/bulut-bilisim-risk-degerlendirmesi-i.html.

- Kossmann, D., Kraska, T., 2011, "Data Management in the Cloud: Promises, State-of-the-art, and Open Questions", Datenbank-Spektrum, Volume 10, Issue 3, 121-129.

- Ortiz, Sixto, 2011, “The Problem with Cloud-Computing Standardization”, IEEE Computer, Volume 44, Issue 7, 13-16, July.

- Pelletier, Stephen, 2009, "What You Need to Know about Cloud Computing”, Associations Now Magazine, (online), December, http://www.asaecenter.org/Resources/ANowDetail.cfm?ItemNumber=46319.

- Saykol, Ediz, 2014, "On the Economical Impacts of Cloud Computing in Information Technology Industry", Proceedings of 5th International Conference on Eurasian Economies (EECON'14), 83-89, Skopje, Macedonia.

- Saykol, Ediz, Avcı, Ayşe Seçil, 2015, "Pivotal Points in Utilizing Cloud Services in Turkey”, Proceedings of 6th International Conference on Eurasian Economies (EECON'15), 170-175, Kazan, Russia.

- Svantesson, D., Clarke, R., 2010, "Privacy and consumer risks in cloud computing”, Computer Law \& Security Review, Volume 26, Issue 4, 391-397. 